www.nature.com/ejhg

\title{
Novel PKD1 deletions and missense variants in a cohort of Hellenic polycystic kidney disease families
}

\author{
Ioanna Bouba ${ }^{1,3,5}$, Michael Koptides ${ }^{1,5}$, Richard Mean ${ }^{1}$, Constandina-Eleni Costi ${ }^{1}$, \\ Kyproula Demetriou ${ }^{2}$, Ioannis Georgiou ${ }^{4}$, Alkis Pierides ${ }^{2}$, Konstantinos Siamopoulos $^{3}$ and \\ C Constantinou Deltas ${ }^{* 1}$
}

\begin{abstract}
${ }^{1}$ The Cyprus Institute of Neurology and Genetics, Department of Molecular Genetics, Nicosia, Cyprus; ${ }^{2}$ Nicosia General Hospital, Department of Nephrology, Nicosia, Cyprus; ${ }^{3}$ University of Ioannina, Department of Nephrology, Ioannina, Greece; ${ }^{4}$ University of Ioannina, Department of Obstetrics/Gynaecology, Ioannina, Greece
\end{abstract}

The autosomal dominant form of polycystic kidney disease is a very frequent genetically heterogeneous inherited condition affecting approximately $1: 1000$ individuals of the Caucasian population. The main symptom is the formation of fluid-filled cysts in the kidneys, which grow progressively in size and number with age, and leading to end-stage renal failure in approximately $50 \%$ of patients by age 60 . About $85 \%$ of cases are caused by mutations in the PKD1 gene on chromosome 16p13.3, which encodes for polycystin-1, a membranous glycoprotein with $\mathbf{4 3 0 2}$ amino acids and multiple domains. Mutation detection is still a challenge owing to various sequence characteristics that prevent easy PCR amplification and sequencing. Here we attempted a systematic screening of part of the duplicated region of the gene in a large cohort of 53 Hellenic families with the use of single-strand conformation polymorphism analysis of exons 16-34. Our analysis revealed eight most probably disease causing mutations, five deletions and three single amino acid substitutions, in the REJ domain of the protein. In one family, a 3-bp and an 8-bp deletion in exons 20 and 21 respectively, were co-inherited on the same PKD1 chromosome, causing disease in the mother and three sons. Interestingly we did not find any termination codon defects, so common in the unique part of the PKD1 gene. In the same cohort we identified 11 polymorphic sequence variants, four of which resulted in amino acid variations. This supports the notion that the PKD1 gene may be prone to mutagenesis, justifying the relatively high prevalence of polycystic kidney disease. European Journal of Human Genetics (2001) 9, 677-684.

Keywords: polycystic kidney disease; PKD1; mutations; polymorphism; renal disease; end-stage renal failure

\section{Introduction}

It is estimated that about $85 \%$ of autosomal dominant polycystic kidney disease (ADPKD) cases are caused by mutations in the PKD1 gene on chromosome 16p13.3 and the rest are caused by mutations in another gene, PKD2 on chromosome $4 \mathrm{q} 22 .{ }^{1,2}$ A third gene, PKD3, has been suggested

\footnotetext{
${ }^{*}$ Correspondence: C Constantinou Deltas, The Cyprus Institute of Neurology and Genetics, 6, International Airport Avenue, Ayios Dhometios, P. O. Box 23462, 1683 Nicosia, Cyprus; Tel: +357 2392655; Fax: +357 2358237; E-mail: DeltasCo@mdrtc.cing.ac.cy

${ }^{5}$ These two authors contributed equally to this work

Received 12 March 2001; revised 5 June 2001; accepted 7 June 2001
}

by some to be implicated in rare families ${ }^{3}$ and disputed by others. ${ }^{4}$ Polycystic kidney disease is considered one of the most common diseases in the Caucasian population, with a prevalance of about $1: 1000$, and is the most common cause of inherited end-stage renal failure. The cardinal feature is the formation of fluid-filled cysts in the kidneys but other symptoms include hypertension, macroscopic or microscopic hematuria and liver cyst formation, among others. ${ }^{5,6}$ The PKD1 and PKD2 genes have been cloned and sequenced ${ }^{7,8}$ and the encoded proteins, polycystin-1 and -2 , have been hypothesised to participate in signal transduction pathways. Several reports have provided convincing evidence that polycystin- 1 and -2 interact with each other through their 
C-terminal cytoplasmic domains and they produce a regulated $\mathrm{Ca}^{2+}$ permeable cation channel. ${ }^{9-12}$ The molecular pathogenesis may involve the need for a somatic second hit that inactivates the inherited normal PKD allele, thereby leading to cystogenesis. ${ }^{13-16}$ More recent work provided genetic evidence in favour of polycystin- 1 and -2 interaction by showing that in certain cases a somatic second hit in the PKD2 gene may be sufficient to cause disease in ADPKD1 patients, leading to a trans-heterozygous situation. ${ }^{2,17}$ Interestingly, germinal trans-heterozygosity caused by co-inheritance of PKD1 and PKD2 mutations was also reported. ${ }^{18}$

Mutations of all kinds, including small insertions and deletions, missence mutations and termination codons have been characterised in more than 150 families by various laboratories. In most cases each family has its own unique mutation, but in rare occasions the same mutation has been found in more than one non-related family and even in families with different ethnic origin. ${ }^{19-25}$

Factors that have been considered to contribute to the PKD1 gene mutagenesis are the high GC content, the large size, $14-\mathrm{kb}$, of the $\mathrm{mRNA}^{26,27}$ and the presence of the longest known polypyrimidine tract of $2.5-\mathrm{kb}$ around intron $21 .^{28}$ Also, some mutations have been occasionally attributed to gene conversion events, as a result of the presence of at least three other highly homologous genes elsewhere on chromosome $16 .^{29}$

In this study we are presenting the first systematic search for mutations in a large part of the duplicated region of the PKD1 gene in a large cohort of ADPKD Hellenic families from Greece. Surprisingly, most mutations found are novel deletions of triplets, representing single aminoacids, or larger deletions that lead to frameshift defects. On one PKD1 chromosome we identified two deletions on different exons, a 3-bp deletion and an 8-bp deletion, both of which we believe are pathogenic. Also, we identified a plethora of missence and neutral polymorphic variants, once again supporting the notion that this gene has high tolerability of DNA sequence variation.

\section{Materials and methods \\ Clinical information}

DNA samples from 53 Hellenic ADPKD families from Greece (24) and Cyprus (29) were included in this study. For some of them we had linkage analysis data suggesting linkage to the PKD1 gene. For the rest no adequate data were available for linkage analysis, or they were single patient families. However, since $85-90 \%$ of all ADPKD cases are due to mutations in the PKD1 gene, they were included in a systematic screening for mutations in the duplicated region of the PKD1 gene.

\section{DNA extraction and LR-PCR}

DNA extraction was performed using the salting out procedure. $^{30}$ The LR-PCR encompassing exons $23-34$ was performed according to the conditions described. ${ }^{15}$ Briefly, PCR buffer consisted of $20 \mathrm{~mm}$ Tris. $\mathrm{HCl} \mathrm{pH} \mathrm{8.75,} 16 \mathrm{~mm}$ (NH4) ${ }_{2} \mathrm{SO}_{4}$, bovine serum albumin $150 \mu \mathrm{g} / \mathrm{ml}, 8 \%$ glycerol, $2.5 \mathrm{~mm} \mathrm{MgCl}_{2}$ and $100 \mu \mathrm{M}$ each dNTP. DMSO to a final concentration of $7 \%$ and $1.5 \mu \mathrm{l}$ of $2 \mathrm{M}$ Trizma-base were also included in the $100 \mu \mathrm{l}$ PCR reaction mix. A hot-start protocol was used with the enzyme mix added at $80^{\circ} \mathrm{C}$. The enzyme mix consisted of 2.4 units of TaqExpress (GenPak, Brighton, UK) and 0.1 units of pfu polymerase (Stratagene, La Jolla, CA, USA). PCR cycling was as follows: denaturation at $94^{\circ} \mathrm{C}$ for 1 min., 35 cycles of $94^{\circ} \mathrm{C}$ for $8 \mathrm{~s}, 58^{\circ} \mathrm{C}$ for $30 \mathrm{~s}$ and $68^{\circ} \mathrm{C}$ for $16 \mathrm{~min}$. The LR-PCR spanning part of exon 15 to 21 was performed with primers FQF26-TWR2 ${ }^{29}$ (Figure 1).

\section{SSCP analysis, DNA cloning and sequencing}

Exon-by-exon screening for mutations was performed on the LR-PCR products by SSCP analysis. ${ }^{15,31}$ Amplification was performed with TaqExpress (GenPak, Brighton, UK). LR-PCR products were diluted $1: 1000$ and $1 \mu \mathrm{l}$ of this was used as a template for all subsequent PCR reactions. This dilution was sufficient to exclude detectable genomic carry-over contamination, since amplification of exons $43-44$ with primer pair 3A3C1/E44R failed. ${ }^{15}$ Sequences of primers and conditions for PCR amplification of the region covering exons 16-21, are shown in Table 1. Primers used for LR-PCR and for PCR-SSCP analysis of sequences in exons 23-46 were published previously. ${ }^{15,31,32}$ Two different conditions were used for SSCP analysis: gels with 29: 1 acrylamide:bisacrylamide were electrophoresed either at $1200 \mathrm{~V}$ for $2.5 \mathrm{~h}$ in a $4^{\circ} \mathrm{C}$ cold room or at very low power, $1 \mathrm{~W}$, at room temperature for $17 \mathrm{~h}$. The abnormal PCR fragments were sequenced on a freshly amplified specific PCR product using the Sequenase PCR sequencing kit from USB (Amersham, Cleveland, Ohio, USA). Sequencing was performed on both strands. For cloning, the PCR product was blunt-end ligated into the Srfl site of the vector pCR-Script $\mathrm{Amp}\left(\mathrm{SK}^{+}\right)$, purchased from Stratagene (La Jolla, California, USA). Ligation and transformation into $E$. coli $\mathrm{DH} 5 \alpha$ cells were performed by standard procedures. Plasmid preparations were made by using a kit from Qiagen (Qiagen GmbH, Hilden, Germany). Sequencing was performed using purified recombinant plasmid DNA. Alternatively, DNA was rapidly extracted by boiling from single colonies, and the insert was specifically amplified by PCR and sequenced directly.

\section{Results and discussion}

Samples from 53 unrelated Hellenic families were screened for mutations in the duplicated part of the PKD1 gene by SSCP analysis. Sequencing of samples showing altered SSCP pattern revealed a number of interesting sequence variants, including clearly pathogenic or putatively pathogenic mutations, in addition to multiple polymorphisms. Eight novel mutations were found in a region of the PKD1 


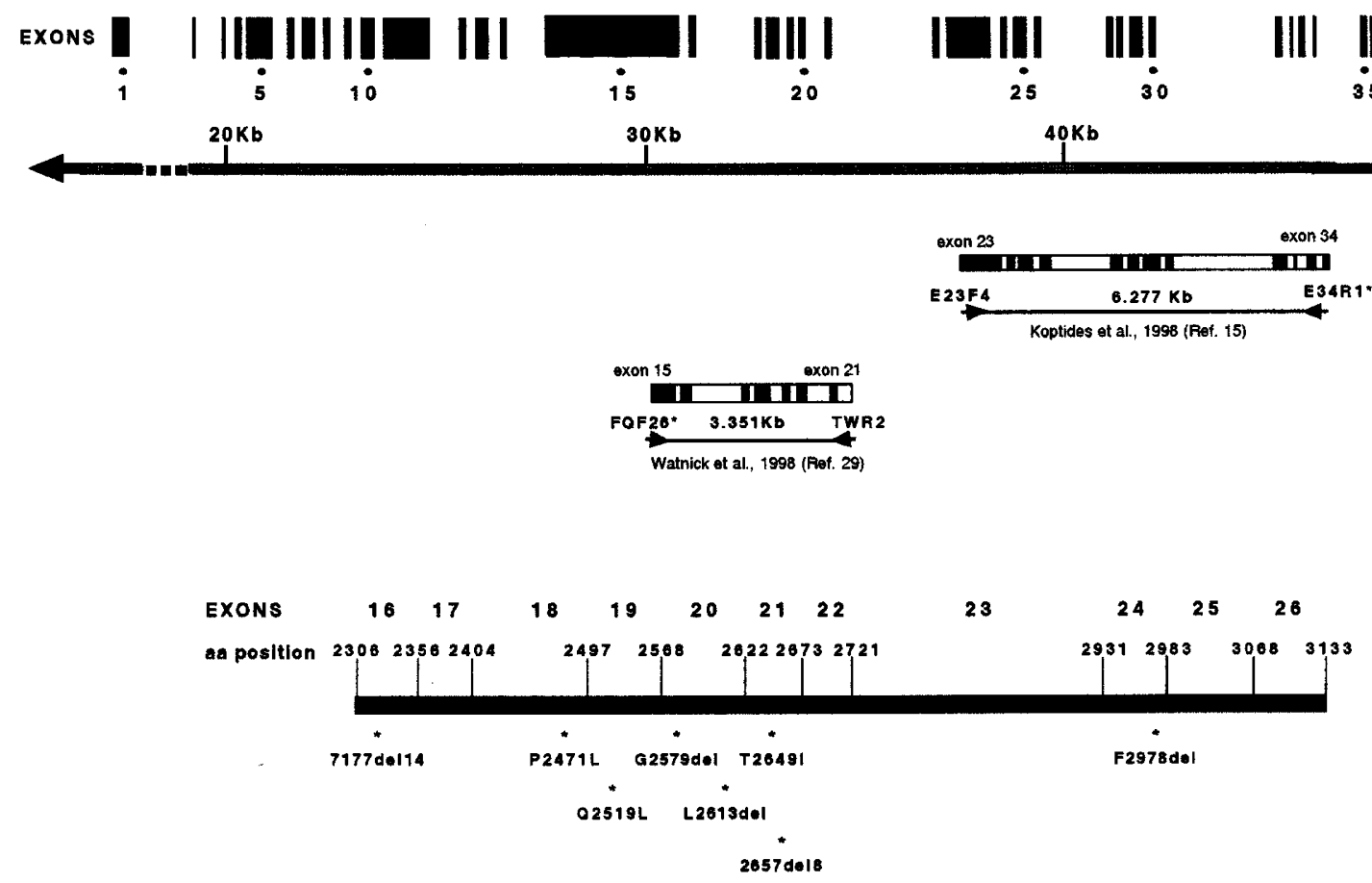

Figure 1 Schematic representation of the PKD1 gene exon-intron structure, showing also the long-range PCR approach used for analysing exons 16-34 (excluding exon 22) by SSCP screening. The lower panel depicts the position of the mutations identified in this work in the Hellenic collection of samples we studied. Notice the slight clustering of mutations in exons 20 and 21 . Only four of about 150 mutations reported in the literature are located in this region.

Table 1 Primer sequences and PCR conditions used in this work for SSCP analysis of PKD1 exons 16-21

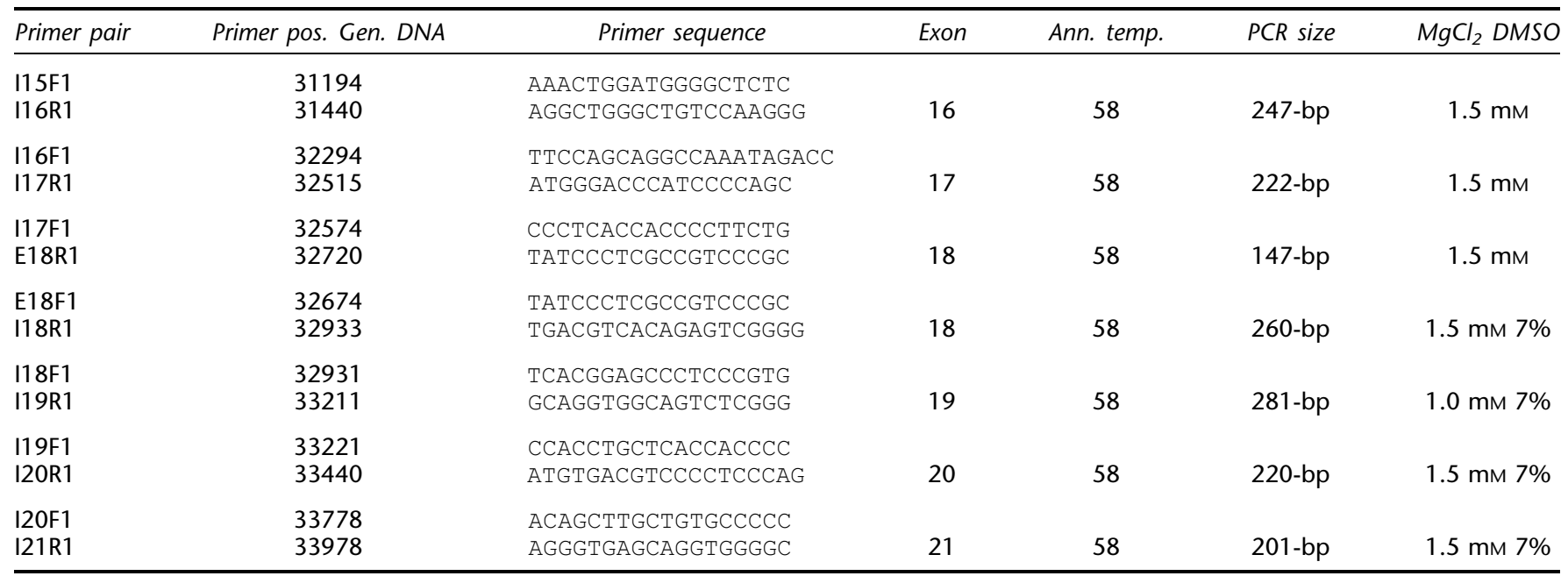

gene that represents $23 \%$ of the coding region, exons $16-$ 21 (Table 2), as well as several polymorphisms, some of which result in aminoacid substitutions (Table 3). Interestingly no nonsense mutation was identified in the duplicated region of the PKD1 gene investigated in this series of experiments.

\section{Mutations}

Deletions

7174-7192del14 (14-bp deleted anywhere therein) SSCP analysis with primers I15F1-I16R1 in exon 16 revealed an abnormal pattern. Subsequent cloning and sequencing revealed a 14-bp deletion which causes a translation frame- 
Table 2 Novel mutations identified in the duplicated region of the PKD1 gene

\begin{tabular}{|c|c|c|c|c|}
\hline Pedigree & Mutations & Exon & Nucleotide change $e^{a}$ & Effect on coding sequence \\
\hline \multicolumn{5}{|l|}{ Deletions } \\
\hline GR-I-32 \& GR-I-38 & 7174del14 or 7179 del14 & 16 & Deletion of $14 \mathrm{bp}$ from 7174 or 7179 & Frameshift \\
\hline GR-I-9 & G2579 del ${ }^{b}$ & 20 & Deletion of 3-bp from 7946 & Deletion of Gly \\
\hline GR-I-35 & L2613del & 20 & Deletion of 3-bp from 8047 & Deletion of leu \\
\hline GR-I-9 & $8183 \mathrm{del} 8^{\mathrm{b}}$ & 21 & Deletion of $8-b p$ from 8183 & Frameshift \\
\hline GR-I-13 & F2978del & 24 & Deletion of 3-bp from 9142 or 9143 & Deletion of Phe \\
\hline \multicolumn{5}{|l|}{ Possible Missense } \\
\hline GR5204 & P2471L & 18 & $\mathrm{C} \rightarrow \mathrm{T}$ at 7623 & Pro $\rightarrow$ Leu at 2471 \\
\hline CY1625 & Q2519L & 19 & $\mathrm{~A} \rightarrow \mathrm{T}$ at 7767 & G1 $n \rightarrow$ Leu at 2519 \\
\hline CY1631 & T2649I & 21 & $\mathrm{C} \rightarrow \mathrm{T}$ at 8157 & $\mathrm{Thr} \rightarrow$ lleu at 2649 \\
\hline
\end{tabular}

a : Nucleotide numbering is according to Hughes et al (1995). ${ }^{b}$ : These deletions occurred on the same chromosome.

Table 3 Polymorphisms identified in the duplicated region of the PKD1 gene during this work

\begin{tabular}{|c|c|c|c|c|}
\hline Polymorphisms & Exon & Nucleotide change $^{a}$ & Effect on coding sequence & References \\
\hline $7376 \mathrm{~T} / \mathrm{C}$ & 17 & $\mathrm{~T} \rightarrow \mathrm{C}$ at 7376 & None & Present work; 43 \\
\hline $7652 \mathrm{C} / \mathrm{T}$ & 18 & $\mathrm{C} \rightarrow \mathrm{T}$ at 7652 & None & Present work; 43 \\
\hline $7919 \mathrm{~T} / \mathrm{C}^{\mathrm{b}, \mathrm{c}}$ & 20 & $\mathrm{~T} \rightarrow \mathrm{C}$ at 7919 & None & Present work; 43 \\
\hline $\mathrm{T} / \mathrm{M} 2582^{\mathrm{b}, \mathrm{c}}$ & 20 & $\mathrm{C} \rightarrow \mathrm{T}$ at 7956 & Thr or Met at 2582 & Present work \\
\hline $8002 \mathrm{~A} / \mathrm{G}^{\mathrm{b}, \mathrm{c}}$ & 20 & $A \rightarrow G$ at 8002 & None & Present work \\
\hline$H / R 2638$ & 21 & $A \rightarrow G$ at 8124 & His or Arg at 2638 & Present work; 43 \\
\hline D/N 2972 & 24 & $\mathrm{G} \rightarrow \mathrm{A}$ at 9125 & Asp or Asn at 2972 & Present work \\
\hline $9406 \mathrm{G} / \mathrm{C}^{\mathrm{d}}$ & 25 & $\mathrm{G} \rightarrow \mathrm{C}$ at 9406 & None & Present work; 21,25 \\
\hline F/L 3066 & 25 & $\mathrm{~T} \rightarrow \mathrm{C}$ at 9407 & Phe or Leu at 3066 & Present work; 21,25 \\
\hline $9481 \mathrm{C} / \mathrm{T}$ & 26 & $\mathrm{C} \rightarrow \mathrm{T}$ at 9481 & None & Present work; 25 \\
\hline $9541 \mathrm{~T} / \mathrm{C}^{\mathrm{c}}$ & 26 & $\mathrm{~T} \rightarrow \mathrm{C}$ at 9541 & None & Present work; 25 \\
\hline
\end{tabular}

${ }^{a}$ : Nucleotide numbering is according to Hughes et al (1995). ${ }^{b}$ : These nucleotide changes occurred on the same chromosome. ${ }^{c}$ : These nucleotide changes are present in Homologous Genes represented by the hybrid cell line P-MWH2A. ${ }^{\text {: }}$ These nucleotide changes occurred on the same chromosome and individual's homozygous for these polymorphisms were observed during this work.

shift leading to putative incorporation of 93 novel aminoacids before reaching a translation STOP codon. It is difficult to define the exact nucleotide position where the deletion appeared, owing to the fact that this deletion is surrounded by a 5-bp direct repeat sequence, CACGG (Figure 2). It is interesting that this nucleotide stretch occurs 32 times within the entire PKD1 coding cDNA, when based on statistics it is expected to occur only 12 times. This may partly be explained by the general GC richness of the gene (67.2\% GC). This deletion was present in two apparently unrelated families originating from the Ioannina prefecture in the north-west of Greece. No haplotype analysis could be performed.

7946del3 (G2579del), 8047del3(L2613del), 9142del3 or 9143del3 (F2978del) An altered SSCP pattern was obtained in exon 20. Sequencing revealed a 3-bp deletion which corresponds to amino acid Gly at codon 2579 (G2579del). Heteroduplex analysis on LR-PCR products obtained from the available mother and the three children, revealed that all had inherited the same deletion, co-segregating with the disease. Also, these same patients had inherited another variation, 8183del8 (see below). The G2579del occurred within a 10-bp imperfect direct repeat (Figure 3). Another 3-bp deletion was identified in exon 20 of a separate single patient, which resulted in the deletion of Leu at codon 2613. And thirdly, a 3-bp deletion was detected in exon 24 of a mother and offspring, abolishing amino acid Phe at codon 2978. In these three cases of single aminoacid deletions, although tempting to implicate them in disease development, in the absence of functional studies and being unable to screen the entire sequence of the PKD1 gene, the data should be interpreted with caution. At the same time, in favour of them being pathogenic and not neutral polymorphisms, is their presence in single families among 53 tested here and among many others tested in other laboratories. It should be mentioned that F2978del occurred within a polypyrimidine-rich 23 -bp sequence ( $87 \% \mathrm{CT}$ ). Within the same 23-bp stretch another 9-bp deletion was reported as a somatic mutation in a renal cyst of a PKD1 patient. ${ }^{2}$ Such polypyrimidine-rich sequences may represent mutational hotspots due to potential formation of triple helical conformations. ${ }^{33,34}$

8183del8 (Frameshift after 2657) In the same family of four clinically affected members who carried the G2579del mutation, a 8-bp deletion in exon 21 was also identified. Cloning and sequencing experiments showed that the two 

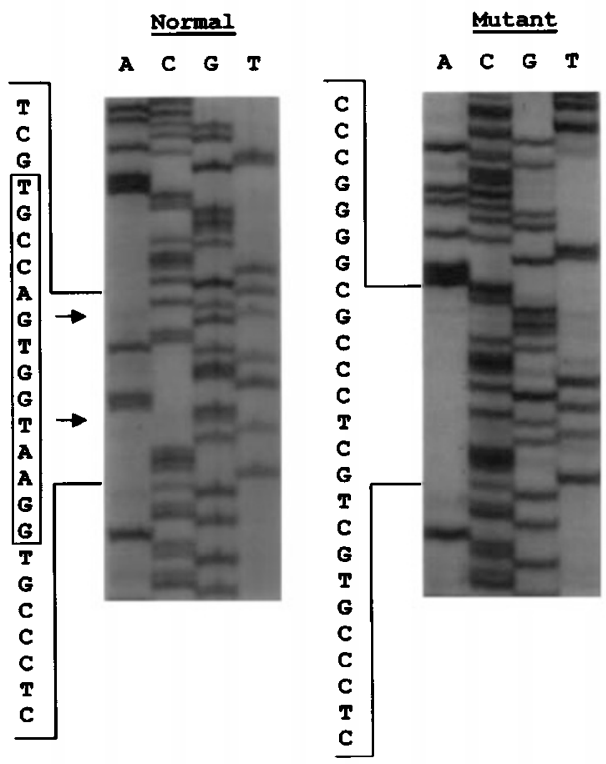

$\underline{\mathbf{B}}$ wild type: GGG AGC AGC ACG GTC ACC ATT CCA CGG GAG CGG
Gly Ser Sor Thr Val Thr Ile Pro Arg Glu Arg

Mutant: GGG AGC AGC ACG GGA GCG GCT GGC GGC TGG CGT Gly Ser Ser Thr GIY Ala Ala GIy Gly Trp Arg

Figure 2 Nucleotide sequence around the region of the mutation $7174-7192$ del14 in exon 16. Antisense sequences were obtained after cloning of PCR products and multiple normal and mutant clones were analysed (A). The 14 residues deleted in the mutant PKD1 allele are boxed and denoted by arrows. The deletion occurred anywhere within the boxed 19-bp sequence (B). Notice that the boxed sequence is flanked by a direct 5-bp repeat (underlined). Interestingly the CACGG sequence is present 32 times within the coding region of PKD1 whereas statistically it is expected only 12 times.

deletions were in cis position on the chromosome that was inherited from the affected mother (Figure 3). This 8-bp deletion is predicted to result in a translation frameshift after aminoacid 2657 and lead to a novel stop codon 483-bp downstream. Based in its nature 8183 del 8 is most likely a pathogenic defect. To our knowledge this is the first time that two putative pathogenic changes seen in cis position, are most probably not the result of gene conversion, as none was present on the homologous genes tested on hybrid cell line PMWH2A. ${ }^{20}$ An unusual pattern of mutation in the PKD1 was previously reported and gene conversion was considered a likely event. ${ }^{21}$ Similar findings have been reported for the CFTR gene, mutations in which are responsible for Cystic Fibrosis and for the MEFV gene, mutations in which are responsible for Familial Mediterranean Fever. ${ }^{35}$ Judging from
A
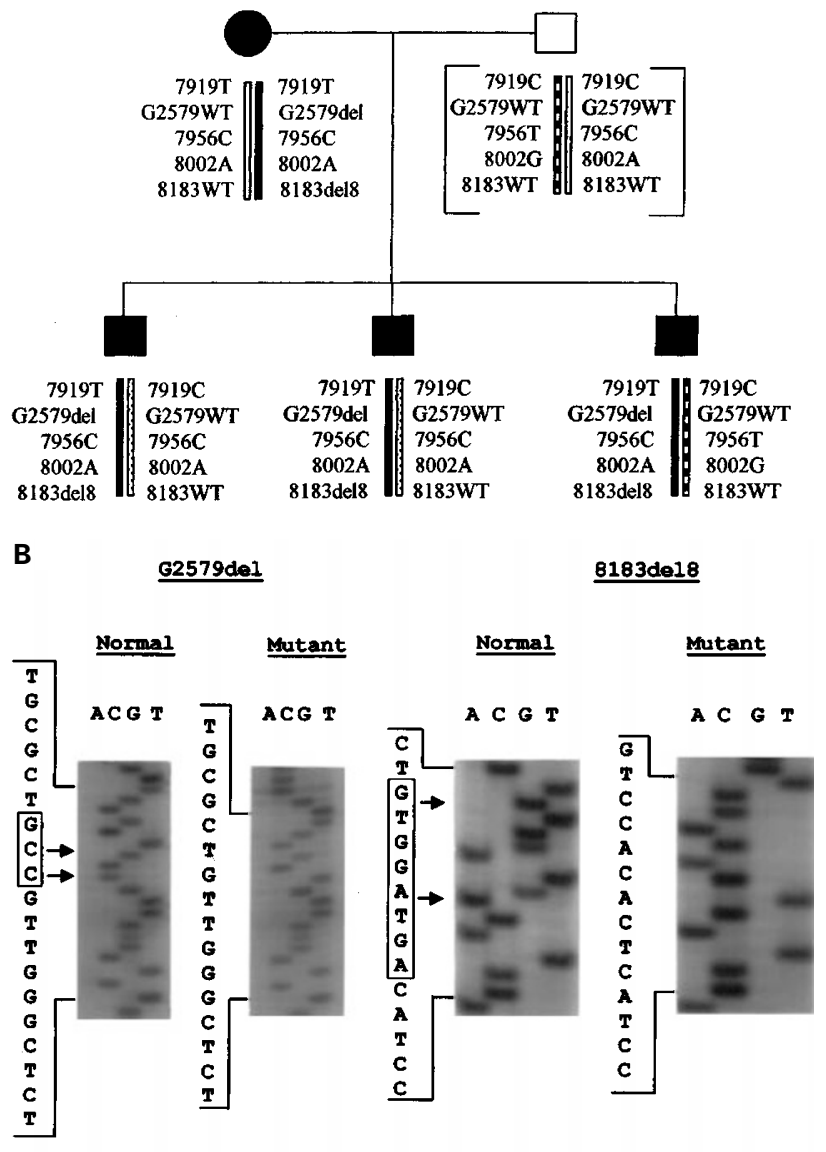

c

Wild Type: cCa gag CoC aAc GGC agc gCa acG gGg Pro Glu Pro Asn GIY Ser Ala Thr Gly

Mutant:

CCA GAg CcC aAC AGC gCa ACg gGg CTC Pro Glu Pro Asn Ser Ala Thr Gly Lou

Figure 3 Pedigree of family Gr-I-9 showing the inheritance of polymorphic PKD1 haplotypes encompassing mutations G2579del and 8183del8. Both mutations are in cis position in exons 20 and 21 respectively. All three children were shown to be affected clinically. Father's haplotypes are deduced (A). Shown are also antisense sequenses of normal and mutant clones containing the 3-bp (B, left) and the 8-bp (B, right) deletions. The two mutations were present on the same cloned fragment carrying exons $20-21$, obtained with PCR primers I19F1/I21R1 (Table 1). The deleted nucleotides are boxed and denoted by arrows (B). The 3-bp deletion G2579del occurred within a region that contained a direct 10 -bp imperfect repeat (underlined, C). In the 10-bp repeat the residue at position 4, was either cytosine or guanosine and are shown with lowercase letters (C). 
the PKD1 gene's structural features, perhaps we should not be surprised by this finding. We anticipate that as technology improves and screening for mutations in this gene becomes easier, more such cases will be found and reported.

\section{Possible missense mutations}

P2471L This mutation (7623C- $>$ T) affects amino acid 2471 in exon 18 by replacing Pro, an iminoacid with cyclic structure which can cause bends on proteins, with Leu, an aliphatic non-polar amino acid. It creates a $B b v I$ restriction site. The 260-bp PCR product of primer-pair E18F1/I19R1 is digested by $B b v \mathrm{I}$ into fragments 130, 65 and 34-bp in the presence of the $\mathrm{T}$ allele and into 164 and 65-bp in the presence of the $\mathrm{C}$ allele. The proline residue is conserved in murine and fugu genomes ${ }^{36}$ (GenBank accession number AF013614), suggesting that this may indeed be the causative defect. This is the only substitution mutation presented in this work that occurred at a CpG dinucleotide, which is known to be prone to this kind of transition. ${ }^{37}$

Q2519L A transversion A-> T at position 7767 of exon 19 leads to a substitution of Leu for Gln. The non-charged but polar hydrophilic amino acid Gln is substituted by the nonpolar hydrophobic Leu. Gln is conserved in murine, but substituted by Asp in fugu genome. This amino acid substitution was present in both affected mother and son in this small family, CY1625, but was absent from another 52 ADPKD unrelated patients. However, the small size of the family does not exclude the possibility that this may represent a private polymorphism and not a causative mutation.

T2649I Sequencing revealed a C- $>$ T substitution at nucleotide position 8157 (Aㄷ- $>$ ATT), changing the amino acid Thr to Ileu at position 2649, in exon 21. It resulted to the replacement of a hydrophilic polar by a hydrophobic nonpolar amino acid. Thr is conserved in murine but is substituted by Ala in fugu.

There is as yet no functional test for the polycystin-1 protein. Therefore, missense mutations, and especially in small families, cannot always be regarded with certainty as the causative defects. However, these variants can be useful when presymptomatic or prenatal diagnosis is attempted by DNA linkage analysis. As regards the deletion mutations, which all occur in the extracellular REJ domain of polycystin1 , it is reasonable to hypothesise that they may interfere with the anchoring of the protein in the plasma membrane and the hypothesised signal transduction.

\section{Polymorphisms}

Eleven intragenic polymorphisms were found, representing neutral or coding variants (Table 3 ). Seven polymorphisms, some of them common, represented silent changes at positions Leu 2389 (7376T/C, heterozygosity 31.5\%), Leu 2481 (7652T/C, heterozygosity 29.6\%), Leu 2570 (7919T/C, heterozygosity 14.8\%), Pro 2597 (8002A/G), Val 3090 (9481C/T), Pro 3110 (9541 T/C). An interesting polymorphism in exon 25 was the result of a substitution of the GT dinucleotide by CC, at positions 9406-9407. This variation affects two adjacent codons, amino acids 3065-3066, that are occupied by either Val-Phe or Val-Leu. It is apparently common, as homozygous individuals were identified with either genotype. ${ }^{21,25}$ Another three missense variants were T/ M2582 (7956C/T), H/R2638 (8124A/G), D/N2972 (9125G/A). In the same family (Gr-I-9) with the three and eight nucleotide deletions described above, a polymorphic haplotype of three polymorphisms in exon 20 was detected, 7919C, 7956T, 8002G. It was obvious that this haplotype was inherited from the healthy parent, and is also present as such in the homologous gene sequences we tested, raising the possibility of gene conversion as a mechanism for their generation (Figure 3A).

In conclusion, our findings of 19 mutations or variations in the 53 Hellenic families investigated here, confirm previous suggestions that the PKD1 gene is prone to mutations. ${ }^{29,34,38}$ De novo mutations reported by several groups confirm this hypothesis. ${ }^{24,25,39}$ The great rarity of some mutations and their confinement within certain families suggests also that they must have occurred very recently. In many cases multiple polymorphisms have been detected on same alleles, being in linkage disequilibrium. ${ }^{40}$ This high variability has been attributed to the high GC content of the gene, the presence of the longest known polypyrimidine track $(2.5-\mathrm{kb})$ and the presence of at least three highly homologous genes that have been shown to be responsible for gene conversion events. $^{29}$ Our data suggest that other features of the PKD1 gene such as random repeats and short CT-rich sequences scattered throughout the gene may contribute to mutagenesis (Figures 2 and 3).

The screening for mutations in this gene is still difficult, primarily because of the very long coding sequence, which prevents one from offering DNA analysis in a routine clinical setup. In this study we identified mutations only in $13 \%$ of our patients by screening a region that represents $23 \%$ of the PKD1 coding sequence. This discrepancy may be explained by the weakness of our approaches, as SSCP is not a $100 \%$ success method. Also, it may be partly explained by the fact that some of the patients belonged to very small families which could not be distinguished, either by clinical or molecular methods, from a PKD2 related disease. Interestingly, approximately 150 PKD1 mutations have been reported thus far $24,39,41$ (Cardiff PKD1 mutation database http://archive.uwcm.ac.uk/uwcm/mg/search/120293.html) only four of which are located in exons 20, 21, whereas four out of eight reported here and four out of 13 identified in Hellenic families altogether, are also located in these exons (Figure 1). Our findings partly support the early hypothesis that clustering of mutations was expected near the intron 21 polypyrimidine tract. 
We wish to emphasise that this is the first study describing a systematic screen for mutations in a large collection of polycystic kidney disease samples from Greece. Finally, we would like to believe that the characterisation of molecular defects and polymorphisms will allow the delineation of polycystin-1 functional domains and may lead to a genotype/ phenotype correlation. At the present time, only the last 112 C-terminal residues of polycystin-1 have been convincingly shown to be involved in the regulation of a calcium channel, most probably through interaction with polycystin-2. This explains why mutations as far C-terminal as C4086X and others, ${ }^{19,31,42}$ which result in removing the crucial polycystin1 fragment that is implicated in regulating a cation channel, ${ }^{12}$ result also in cyst formation and disease development.

\section{Acknowledgments}

This work was funded partly by the Cyprus Ministry of Health and the Cyprus Kidney Association.

\section{References}

1 Peters DJ, Sandkuijl LA: Genetic heterogeneity of polycystic kidney disease in Europe; In: Breuning MH, Devoto M, Romeo G, (eds): Contrib Nephrol. Basel, Karger, 1992, vol 97, pp 128-139.

2 Koptides M, Deltas CC: Autosomal Dominant Polycystic Kidney Disease: Molecular Genetics and Molecular Pathogenesis. Hum Genet 2000; 107: 115-126.

3 Daoust MC, Reynolds DM, Bichet DG, Somlo S: Evidence for a third genetic locus for autosomal dominant polycystic kidney disease. Genomics 1995; 25: $733-736$.

4 Paterson AD, Pei Y: PKD3-to be or not to be? Nephrol Dial Transplant 1999; 14: $2965-2966$.

5 Dalgaard OZ: Bilateral polycystic disease of the kidneys: a follow-up of two hundred and eighty-four patients and their families. Acta Med Scand 1957; 328: 1-251.

6 Gabow PA: Autosomal dominant polycystic kidney disease. $N$ Engl J Med 1993; 329: 332-342.

7 European Polycystic Kidney Disease Consortium: The polycystic kidney disease 1 gene encodes a $14 \mathrm{~kb}$ transcript and lies within a duplicated region on chromosome 16. Cell 1994; 77: 881-894.

8 Mochizuki T, Wu G, Hayashi T et al: PKD2, a gene for polycystic kidney disease that encodes an integral membrane protein. Science 1996; 272: 1339-1342.

9 Qian F, Germino EJ, Cai Y et al: PKD1 interacts with PKD2 through a probable coiled-coil domain. Nature Genet 1997; 16: $179-183$.

10 Tsiokas L, Kim E, Arnould T et al: Homo- and heterodimeric interactions between the gene products of PKD1 and PKD2. Proc Natl Acad Sci USA 1997; 94: 6965-6970.

11 Hanaoka K, Qian F, Boletta A et al: Co-assembly of polycystin-1 and -2 produces unique cation-permeable currents. Nature 2000; 408: $990-994$

12 Vandorpe DH, Chernova MN, Jiang L et al: The cytoplasmic carboxy-terminal fragment of polycystin-1 (PKD1) regulates a $\mathrm{Ca}^{2+}$-permeable cation channel. J Biol Chem 2000; 276: 4093 4101.

13 Qian F, Watnick TJ, Onuchic LF et al: The molecular basis of focal cyst formation in human autosomal dominant polycystic kidney disease type 1. Cell 1996; 87: 979-987.

14 Brasier JL, Henske EP: Loss of the polycystic kidney disease (PKD1) region of $16 \mathrm{p} 13$ in renal cyst cells supports a loss-offunction model for cyst pathogenesis. J Clin Invest 1997; 99: 194-199.
15 Koptides M, Constantinides R, Patsalis CP et al: Loss of heterozygocity in polycystic kidney disease with a missense mutation in the repeated region of PKD1. Hum Genet 1998; 103: $709-717$.

16 Koptides M, Hadjimichael C, Koupepidou P et al: Germinal and somatic mutations in the PKD2 gene of renal cysts in autosomal dominant polycystic kidney disease. Hum Mol Genet 1999; 8: 509-513.

17 Koptides M, Mean R, Demetriou K et al: Genetic evidence for a trans-heterozygous model for cystogenesis in autosomal dominant polycystic kidney disease. Hum Mol Genet 2000; 12: $447-$ 452.

18 Pei Y, Paterson AD, Wang KR et al: Bilineal disease and transheterozygotes in autosomal dominant polycystic kidney disease. Am J Hum Genet 2001; 68: 355 - 363.

19 Peral B, San Millan JL, Ong ACM et al: Screening of the 3 ' region of the polycystic kidney disease 1 (PKD1) gene reveals six novel mutations. Am J Hum Genet 1996 58: 86-96.

20 Peral B, Gamble V, Strong C et al: Identification of mutations in the duplicated region of the polycystic kidney disease 1 gene (PKD1) by a novel approach. Am J Hum Genet 1997; 60: 13991310.

21 Watnick TJ, Piontek KB, Cordal TM et al: An unusual pattern of mutation in the duplicated portion of PKD1 is revealed by use of a novel strategy for mutation detection. Hum Mol Genet 1997; 6: $1473-1481$.

22 Roelfsema JH, Spruit L, Saris JJ et al: Mutation detection in the repeated part of the PKD1 gene. Am J Hum Genet 1997; 61: $1044-1052$.

23 Thomas R, McConnell R, Whittacker J et al: Identification of mutations in the repeated part of autosomal dominant polycystic kidney disease type 1 gene, PKD1, by long-range PCR. Am J Hum Genet 1999; 65: 39-49.

24 Phakdeekitcharoen B, Watnick TJ, Ahn C, Whang DY, Burkhart B, Germino GG: Thirteen novel mutations of the replicated region of PKD1 in an Asian population. Kidney Int 2000; 58: $1400-1412$.

25 Koptides M, Mean R, Demetriou K et al: Screening of the PKD1 duplicated region reveals multiple single nucleotide polymorphisms and a de novo mutation in Hellenic polycystic kidney disease families. Hum Mut 2000; 16: 176 (Online).

26 American PKD1 Consortium: Analysis of the genomic sequence for the autosomal dominant polycystic kidney disease (PKD1) gene predicts the presence of a leucine-rich repeat. Hum Mol Genet 1995; 4: $575-582$.

27 Hughes J, Ward CJ, Peral B et al: The polycystic kidney disease 1 (PKD1) gene encodes a novel protein with multiple cell recognition domains. Nature Genet 1995; 10: 151-160.

28 Van Raay TJ, Burn TC, Connors TD et al: A $2.5 \mathrm{~kb}$ polypyrimidine tract in the PKD1 gene contains at least 23 H-DNAforming sequences. Microb Comp Genomics 1996; 1: 317-327.

29 Watnick TJ, Gandolph MA, Weber H et al: Gene conversion is a likely cause of mutation in PKD1. Hum Mol Genet 1998; 7: 1239_ 1243.

30 Miller SA, Dykes DD, Polesky HF: A simple salting out procedure for extracting DNA from human nucleated cells. Nucleic Acids Res 1988; 16: 1215.

31 Neophytou P, Constantinides R, Lazarou A et al: Detection of a novel nonsense mutation and an intragenic polymorphism in the PKD1 gene of an Autosomal Dominant Polycystic Kidney Disease Cypriot family. Hum Genet 1996; 98: 437-442.

32 Neophytou P, Constantinides R, Girginoudis P et al: Identification of novel and recurrent mutations in the polycystic kidney disease 1 gene (PKD1) by single strand conformation analysis. Balkan J Med Genet 1998; 1: 149-159.

33 Wang G, Seidman MM, Glazer MP: Mutagenesis in mammalian cells induced by triple helix formation and transcription coupled repair. Science 1996; 271: 802-880. 
34 Germino GG: Autosomal dominant polycystic kidney disease: A two-hit model. Hosp Pract 1997; 32: 81-92.

35 Samuels J, Askentijevich I, Torosyan Y et al: Familial Mediterranean Fever at the millennium. Clinical spectrum, ancient mutations, and a survey of 100 American referrals to the National Institute of Health. Medicine 1998; 77: 268 - 297.

36 Lohning C, Nowicka U, Frischauf A-M: The mouse homolog of the PKD1: sequence analysis and alternative splicing. Mamm Genome 1997; 8: 307-311.

37 Cooper DN, Krawczak M, Antonarakis SE: The nature and mechanisms of human gene mutation. In: Scriver $C$, Beaudet AL, Sly WS, Valle D, (eds): Metabolic and molecular basis of inherited disease. McGraw-Hill, New York, 1995, pp 259-291.

38 International Polycystic Kidney Disease Consortium: Polycystic kidney disease: the complete structure of the PKD1 gene and its protein. Cell 1995; 81: 289-298.

39 Rossetti S, Strmecki L, Gamble V et al: Mutation analysis of the entire PKD1 gene: Genetic and diagnostic implications. Am J Hum Genet 2001; 68: 46-63.
40 Constantinides R, Xenophontos S, Neophytou P, Nomura S, Pierides A, Constantinou Deltas C: New aminoacid polymorphism, Ala/Val.4058, in exon 45 of the polycystic kidney disease 1 gene: Evolution of alleles. Hum Genet 1997; 99: 644-647.

$41 \mathrm{Wu}$ G, Somlo S: Molecular genetics and mechanism of autosomal dominant polycystic kidney disease. Mol Genet Metab 2000; 69: $1-15$.

42 Perrichot RA, Mercier B, Simon PM et al: DGGE screening of PKD1 gene reveals novel mutations in a large cohort of 146 patients. Hum Genet 1999; 105: 231-239.

43 Watnick T, Phakdeekitcharoen B, Johnson A et al: Mutation detection of PKD1 identifies a novel mutation common to three families with aneurysms and/or very-early-onset disease. Am J Hum Genet 1999; 65: 1561 - 1571. 